\title{
Mentoring and Diversity
}

\section{Citation}

Athey, Susan, Christopher Avery, and Peter Zemsky. 2000. “Mentoring and Diversity.” American Economic Review 90 (4): 765-86. https://doi.org/10.1257/aer.90.4.765.

\section{Permanent link}

http://nrs.harvard.edu/urn-3:HUL.InstRepos:41426685

\section{Terms of Use}

This article was downloaded from Harvard University's DASH repository, and is made available under the terms and conditions applicable to Other Posted Material, as set forth at http:// nrs.harvard.edu/urn-3:HUL.InstRepos:dash.current.terms-of-use\#LAA

\section{Share Your Story}

The Harvard community has made this article openly available.

Please share how this access benefits you. Submit a story.

Accessibility 


\title{
American Economic Association
}

\author{
Mentoring and Diversity \\ Author(s): Susan Athey, Christopher Avery and Peter Zemsky \\ Source: The American Economic Review, Vol. 90, No. 4 (Sep., 2000), pp. 765-786 \\ Published by: American Economic Association \\ Stable URL: http://www.jstor.org/stable/117307 \\ Accessed: 26/07/2013 16:24
}

Your use of the JSTOR archive indicates your acceptance of the Terms \& Conditions of Use, available at http://www.jstor.org/page/info/about/policies/terms.jsp

JSTOR is a not-for-profit service that helps scholars, researchers, and students discover, use, and build upon a wide range of content in a trusted digital archive. We use information technology and tools to increase productivity and facilitate new forms of scholarship. For more information about JSTOR, please contact support@jstor.org. 


\title{
Mentoring and Diversity
}

\author{
By Susan Athey, Christopher Avery, And Peter Zemsky*
}

\begin{abstract}
We study how diversity evolves at a firm with entry-level and upper-level employees who vary in ability and "type" (gender or ethnicity). The ability of entry-level employees is increased by mentoring. An employee receives more mentoring when more upper-level employees have the same type. Optimal promotions are biased by type, and this bias may favor either the minority or the majority. We characterize possible steady states, including a "glass ceiling," where the upper level remains less diverse than the entry level. A firm may have multiple steady states, whereby temporary affirmative-action policies have a long-run impact. (JEL J71, J41, D20)
\end{abstract}

The related topics of workplace diversity and of biased hiring and promotion decisions engender impassioned discourse in contemporary American society. Some complain that affirmative-action policies lead firms to discriminate against white males. Others counter that the historic domination of management by white males puts other groups at a disadvantage, arguing that the underlying nature of the workplace inherently favors those who are from similar backgrounds as their managers. They point to the fact that in many industries and occupations, women and minorities have moved only slowly into upper-level positions, despite the fact that the labor pool and lower levels of the work force have been diverse for some time. ${ }^{1}$ This

\footnotetext{
* Athey: Department of Economics, Massachusetts Institute of Technology, 50 Memorial Drive, Cambridge, MA 02142; Avery: Kennedy School of Government, Harvard University, Cambridge, MA 02138; Zemsky: INSEAD, Boulevard de Constance, Fountainbleau Cedex 77305, France. We thank three anonymous referees for insightful comments, which greatly improved the paper. We are also grateful to Jim Baron, Jeremy Bulow, Peter Diamond, Joshua Gans, Ed Lazear, Michael Kremer, Bill Lovejoy, Meg Meyer, Bob Marshall, John Roberts, Scott Stern, Bob Wilson, and seminar participants at Oxford, Stanford, and Yale Universities for helpful discussions. Any errors remain our own. The authors acknowledge support from the State Farm Companies Foundation and the National Science Foundation (Athey, SBR-9631760). Athey and Avery are also grateful to the Cowles Foundation at Yale for hospitality and support while this work was being completed.

${ }^{1}$ Large gaps exist between the proportion of women at lower levels and higher levels of large corporations (Ann Morrison and Mary Ann Von Glinow, 1990) and law firms (Stephen Spurr, 1990), and also to a lesser extent in academic economics departments (Robin Bartlett, 1997). In many occupations, the 1980 Census revealed a higher pro-
}

phenomenon has been referred to as the "glass ceiling."

Some firms have made active efforts to reshape themselves, instituting affirmative-action programs and hiring diversity managers. For example, IBM is known for its affirmative-action program, whereas American Airlines, AT\&T, Colgate-Palmolive Corp., DuPont Corp., and Pacific Bell have instituted diversity programs. ${ }^{2}$ Other firms aggressively fight external constraints on their staffing decisions. It is difficult to reconcile this heterogeneity among firms without specifying why firms care about diversity.

This paper develops a model to analyze the relationship between the diversity of a firm's upper level and its internal promotion policies. We consider the dynamic problem faced by a firm in choosing which of its lower-level employees to promote as its existing upper-level workers retire. Employees are characterized by an initial ability for upper-level work and by a type, which can be interpreted as gender, ethnicity, cultural background, personality type, or even skill set (e.g., operations versus marketing skills for managers, theorists versus empiricists for academics). We assume that the lower level is split evenly between two types. We refer to the type that has most of the upper-level positions as the "majority" and the other type as the "minority."

portion of women, Blacks, and Hispanics in "employee" positions than in "supervisor" positions (Donna Rothstein, 1997).

${ }^{2}$ The Wall Street Journal (August 9, 1991 p. B1). 
Employees in our model augment their initial ability by acquiring specific human capital in mentoring interactions with upper-level employees. We interpret mentoring broadly, including activities such as information sharing, informal teaching, or career advice provided by more senior workers. A critical assumption in our model is that mentoring is "type-based," in that an entry-level employee acquires more human capital from mentoring when the firm has more upper-level employees who match her type.

Type-based mentoring is consistent with evidence from a variety of sources. Psychologists and sociologists have documented that mentoring relationships within firms are more likely to form between members of the same group. ${ }^{3}$ Herminia Ibarra (1992) demonstrates that the structure of social networks depends on gender and race. More generally, communication, and thus mentoring, may be more natural and more effective when people share common interests (such as sports), cultural experiences, language, or when people have significant interactions in a community outside the workplace. ${ }^{4}$ Type-based mentoring has also been highlighted in accounts of professional partnerships. For example, a $\mathrm{Na}$ tional Law Journal article ${ }^{5}$ reported,

Despite women's progress, the partnership ranks are 86.4 percent male. This puts women at a disadvantage when it comes to mentoring, the most important factor in becoming a partner. Often it's harder for women to find a mentor, because the massive majority of partners are men, and men tend to be more comfort-

\footnotetext{
${ }^{3}$ Raymond Noe (1988) and Morrison and Von Glinow (1990) review the theory and evidence in favor of typebased mentoring. More recently, George F. Dreher and Taylor H. Cox (1996) document such differences in a survey of MBA graduates. Rosabeth Kanter's classic (1977) analysis of gender roles in organizations takes a similar view, observing that "numbers-proportional representation are important not only because they symbolize the presence or absence of discrimination but also because they have real consequences for performance" (p. 6).

${ }^{4}$ In a related set of historical examples, Avner Greif $(1993,1994)$ shows that cooperation in trading relationships can at times be more easily sustained between members of an extended family or community.

5 "Women's Progress Slows at Top Firms," May 6, 1996 p. A1.
}

able mentoring other men. Rainmaking and client development-skills typically learned from a mentor-are keys to partnership. ${ }^{6}$

We show that type-based mentoring has significant and sometimes complex effects on optimal promotion policies and on the evolution of diversity at a firm. The direct effect of type-based mentoring is that entry-level employees of the majority type acquire more human capital, and thus firms who base promotions solely on ability promote more majority employees. However, since upper-level diversity affects a firm's profits through the mentoring of future workers, the optimal policy of a forward-looking firm will generally involve promoting workers who do not have the highest ability to influence the evolution of diversity over time.

Our approach suggests that observed differences in promotion decisions result from (i) true productivity differences, which arise (partly) as a result of a firm's past promotion decisions, and (ii) what we call bias in promotions, that is, a decision to pass over some workers with higher ability as part of a long-term plan to move to a desired level of diversity. Whereas much of the political debate about affirmative action and discrimination centers on issues of fairness in the evaluation of individual workers at a given point in time, our model suggests that there are important efficiency considerations, as well, in that firms can benefit from considering today's promotion decisions as an investment in future mentoring.

An important question is whether forwardlooking firms benefit from increasing or

\footnotetext{
${ }^{6}$ Similar claims have been made about the management consulting industry: "The lack of role models and mentors who are black is a problem.... [t] blacks in corporate America-lack of connections, lack of mentors, and the often-present glass ceiling-face blacks in management consulting" (Gautam Prakash, 1995). Similarly, The Wall Street Journal ("Women Make Strides, But Men Stay Firmly in Top Company Jobs," March 29, 1994 p. A1), reporting on the effects of culture in large U.S. companies, argued that "as long as the percentages of male managers remain high, the culture remains mostly male and women say, indifferent or hostile to their advancement. Women say they are ignored, not taken seriously ... [so] there is little chance of breaking through the glass ceiling."
} 
decreasing diversity. In our model, the opportunity cost of a homogeneous upper level is an inability to take advantage of the scarce talent of entry-level minority employees. Since minorities receive relatively little mentoring, even a minority with a very high initial ability may be passed over in favor of a less initially able, but better mentored, majority type. Formally, we show that the scarcer the initial ability (i.e., the faster the initial ability of each type of worker diminishes in the number of that type promoted), the more the firm shifts the bias toward the minority type. Consistent with this analysis, the desire to exploit the talents of an increasingly diverse labor market is often cited by firms that actively promote workforce diversity. ${ }^{7}$

In general, we find that the optimal bias need not favor the minority, even if there are decreasing returns to having more mentors of a given type. Because majority employees are better mentored, their promotion rates can be higher than those of minorities, leading the firm to care more about the effective mentoring of majority than minority employees. As a result, a profit-maximizing firm will bias its promotions to favor increased diversity only if there are sufficiently decreasing returns to mentors of a given type. The extent of decreasing returns required to trigger a bias for the minority depends on the scarcity of initial ability, the retirement rate, and the importance of mentoring.

After characterizing the optimal bias, we consider the effect of type-based mentoring on the long-run level of diversity. We show that diversity of the upper level converges to a steady state, which can range from full diversity to complete homogeneity. Our model can exhibit a

\footnotetext{
${ }^{7}$ For example, Business Week ("White, Male, and Worried," January 31, 1994 pp. 50-55) reported that to some companies, "managing diversity ... is a competitive weapon that helps the company capitalize on its talent pool. The goal: to create a culture that enables all employees to contribute their full potential to the company's success. One way is to groom more qualified women and minorities through active succession planning." Similarly, it has been claimed that companies use diversity programs to "attract and retain the best and the brightest ... giving a headstart in recruiting and managing the workers of tomorrow" (USA Today, "Setting Diversity's Foundation in the Bottom Line," by Del Jones, October 15, 1996 p. 4B).
}

glass ceiling phenomenon, whereby an initially homogeneous management partially diversifies, but the "invisible barrier" of type-based mentoring stalls the progress of minorities before the diversity of the upper level mirrors the lower level. Although in some cases there may be a unique long-run level of diversity, type-based mentoring can naturally lead to multiple steadystate levels of diversity because the gains of minorities are self-reinforcing. As minority representation increases in the upper level, the mentoring disadvantage faced by new minority employees decreases, making it more attractive to promote them. As a result, short-run pressure on a firm to diversify can have long-lasting impact by moving the firm from one steady state to another.

Our model assumes that the lower level of the firm is heterogeneous. This assumption highlights the central trade-off in the model: homogeneity increases the productivity of majority mentoring, but imposes an opportunity cost when the firm passes over scarce minority talent. This model is well suited to address the empirical question of why the upper levels of firms remain homogeneous, even when the lower levels are much more diverse. However, it remains to consider when lower-level heterogeneity is consistent with type-based mentoring. In Section I, we discuss a variety of (unmodeled) labor-market frictions that could give rise to such heterogeneity, and in Section V we discuss an extension of the model in which lower-level diversity is an equilibrium outcome.

Our paper differs from previous theoretical work on discrimination (e.g., Kenneth Arrow, 1973; Stephen Coate and Glenn Loury, 1992; Bradford Cornell and Ivo Welch, 1996; Asa Rosen, 1997) in several ways. First, prior work is best suited for understanding discrimination in hiring rather than in promotion decisions. For example, the assumptions of incomplete or asymmetric information about worker abilities that underlies much prior work are less palatable when an employee has been with a firm for a long period of time. Second, prior work does not consider dynamically optimal policies. Finally, most studies do not unravel the forces that lead to discrimination within a firm, and all prior work deals with a one-on-one relationship between the 
worker and the firm. None formalizes the idea that the current diversity of a firm affects the career paths of new employees. ${ }^{8}$

An important policy implication in the received literature is that affirmative-action policies can actually impede the progress of minorities by reinforcing beliefs that minorities are less qualified (Coate and Loury, 1992). Here, we show that affirmative action may increase diversity in the long run by inducing the firm to shift from one steady-state level of diversity to another. It is important to recognize, however, that our formal analysis involves optimizing behavior without externalities, and hence there are no inefficiencies. Unless the social welfare function includes a taste for diversity, our results do not motivate government constraints on the promotion decisions of firms, such as the short-run pressure for diversity just described. Of course, there are a variety of reasons that a society might care about the diversity of firms. For example, the firms or the agents responsible for promotions might discount the future excessively, perhaps as a result of agency problems. Further, as we discuss in Section VI, firms may not internalize the effect of their promotion decisions on the incentives of workers to acquire human capital. ${ }^{9}$

The paper proceeds as follows. We introduce and discuss the formal model in Section I. Section II characterizes when the optimal bias favors diversity or homogeneity. Section III analyzes how diversity evolves over time. Section IV discusses the empirical implications of the model and provides comparative statics results. Sections V and VI discuss two extensions to the model: a labor market for entry-level

\footnotetext{
${ }^{8}$ Consider, for example, Cornell and Welch (1996) who study "screening discrimination" where employers are better able to evaluate the abilities of job applicants of the same type as themselves. Their theory depends on uncertainty about ability; their firm consists of a single employer and a single worker; and they do not consider forward-looking hiring policies where firms hire minorities today so as to better screen them in the future.

${ }^{9}$ Yet another potential source of inefficiencies is that successful members of a type (i.e., individuals who attain upper-level positions) may serve as "role models" for younger members of their community. This role modeling would be very much like our broad definition of mentoring, except that the human-capital transfers would happen outside of the firm and hence would not be incorporated into firm decision making.
}

employees, and ex ante human capital acquisition. Section VII concludes.

\section{The Model}

A single firm employs a continuum of upperlevel and lower-level employees. ${ }^{10}$ We normalize the measure of upper-level employees to 1 . There are two types of employees, labeled $A$ and $B$. The proportion of upper-level employees of type $A$ in period $t$ is denoted $m^{t}$. In each period a proportion $r \in(0,1]$ of the upperlevel employees retire and must be replaced. Retirement rates are uniform, meaning that $\mathrm{rm}^{t}$ workers of type $A$ and $r\left(1-m^{t}\right)$ workers of type $B$ retire in period $t$. We will typically treat $A$ as the majority and $B$ as the minority in our exposition (i.e., $m^{t} \geq 1 / 2$ ).

The firm operates an internal labor market in which retiring employees are replaced from the lower level, which has an equal number of $A$ 's and $B$ 's. With this assumption we introduce a cost of upper-level homogeneity, which we believe to be quite general: homogeneous firms bear the opportunity cost of passing over talented workers of the opposite type within their organization. There are numerous reasons why the lower levels of organizations are diverse despite type-based mentoring (which introduces a benefit to segregating entry levels by type). For example, firms generally differ in their locations, cultures, and skill requirements, and thus workers may have firm-specific preferences and skills that make it beneficial for a diverse group of employees to work at the same firm. Search costs might reinforce the effects of firm-worker matching. Further, in the present legal and political environment, a variety of nonmarket forces create pressure for diverse lower levels. ${ }^{11}$ Finally, as discussed in Section V, partial diversity of the lower level can arise in equilibrium even without labor-market frictions.

\footnotetext{
${ }^{10}$ In our 1994 Stanford GSB working paper (Athey et al., 1994) we consider a model of type-based mentoring with a finite number of employees where initial ability is stochastic. The qualitative insights of the stochastic model are the same, but the deterministic model analyzed in this paper is more tractable.

${ }^{11}$ Segregated firms may be at risk of government and civil litigation for biased hiring practices. They may also face social pressure to mirror the composition of their local labor markets.
} 
We assume that there are at least $r$ entrylevel workers of each type and that the two pools of lower-level workers are symmetric in their initial abilities. Member $\theta \in[0, r]$ of a pool of entry-level workers has an initial ability $x(\theta)$, which represents the surplus gained by the firm when worker $\theta$ takes an upper-level position. ${ }^{12}$ The function $x(\theta)$ is assumed to be nonincreasing. Thus, the quality of the marginal worker from each pool (weakly) decreases as the firm digs deeper into that pool, representing scarcity of ability for upper-level work. Such scarcity may be especially relevant for jobs in the service sector, jobs requiring education and specialized skills, jobs where "stars" are important (such as academics, entertainment, and sports), and high-level management positions in firms.

A worker's type and initial ability are observed by the employer. Each entry-level employee gains additional (firm-specific) skills required for upperlevel work through mentoring, represented by the function $\mu(\cdot)$, which depends on the proportion of upper-level employees with the same type. ${ }^{13}$ This mentoring function is assumed to be increasing and continuously differentiable. A promoted worker makes an overall (lifetime) contribution to the firm's profit that depends on her type, her initial ability, and the composition of the firm when she is promoted: ${ }^{14}$

$$
s_{A}(\theta, m)=x(\theta)+\alpha \mu(m)
$$

\footnotetext{
${ }^{12}$ There are several potential sources of such a surplus. The worker's ability might be specific to the firm-worker match, it might not be observable by other firms, or there might be frictions in the labor market.

${ }^{13}$ The effectiveness of mentoring does not depend on the ability of upper-level employees. If mentoring depends linearly on the average ability of all upper-level employees, then our analysis is unaffected. However, the analysis is affected if mentoring depends on the average ability of upper-level employees with the same type. We expect that such dependency would reduce the magnitude of the promotion bias because promoting less-able candidates becomes less attractive.

${ }^{14}$ These surplus functions do not include the indirect contribution from future mentoring of other workers; these effects are incorporated into the firm's dynamic programming problem that follows. Further, observe that, although our primary interpretation of mentoring is firm specific, any general skills in mentoring may accrue to the workers. Thus the surplus functions can be interpreted as the benefit to the firm net of wages.
}

and

$$
s_{B}(\theta, m)=x(\theta)+\alpha \mu(1-m),
$$

where $\alpha$ parameterizes the importance of mentoring relative to initial ability. We denote by $z^{t} \in[0, r]$ the measure of the promoted employees in period $t$ who are of type $A ; r-z^{t}$ are then of type $B$. The firm's per-period profit function is the total surplus generated by its new upper-level employees:

$$
\begin{aligned}
\pi(m, z)= & \int_{0}^{z} s_{A}(\theta, m) d \theta \\
& +\int_{0}^{r-z} s_{B}(\theta, m) d \theta .
\end{aligned}
$$

The firm seeks to maximize the discounted sum of its per-period profits by choosing a sequence of promotion policies $\left(z^{1}, z^{2}, \cdots\right)$ to solve the following maximization problem:

$$
\text { (1) } \begin{aligned}
V(m)= & \max _{\left(z^{1}, z^{2}, \cdots\right)} \sum_{t=0}^{\infty} \delta^{t} \pi\left(m^{t}, z^{t}\right) \\
& \text { subject to } m^{t+1}=(1-r) m^{t}+z^{t}, \\
& z^{t} \in[0, r] \quad \text { and } \quad m^{0}=m .
\end{aligned}
$$

Given a value function (which is established to be well behaved for our model in the Appendix), we can define the optimal policy correspondence by

$$
\begin{aligned}
z^{*}(m) & =\{z \in[0, r] \mid V(m) \\
& =\pi(m, z)+\delta V(z+(1-r) m)\}
\end{aligned}
$$

We are interested in how $m^{t}$ evolves over time and in how the firm biases its promotions. We define the unbiased (or myopic) promotion policy $z^{U B}(m)$ implicitly from the equation

$$
s_{A}\left(z^{U B}, m\right)=s_{B}\left(r-z^{U B}, m\right),
$$




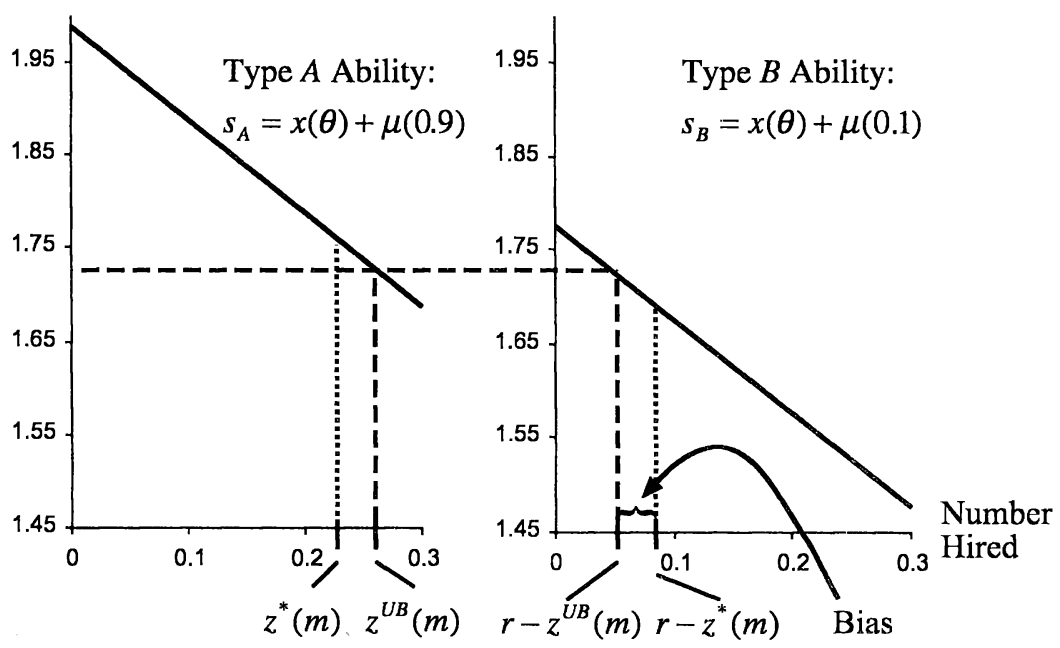

Figure 1. Surplus Functions and Promotion Policies

Notes: As indicated with the horizontal line, unbiased promotions equalize final abilities across the two types. The optimal promotion policy entails a bias in favor of type $B$. Parameters are given in Example 1 with $\delta=0.95$.

which equalizes the contribution of the marginal promoted employee of each type. ${ }^{15}$

We can now define the bias in the firm's optimal promotion policy as

$$
b(m)=z^{U B}(m)-z^{*}(m) .
$$

A positive bias $b(m)>0$ is then a bias in favor of type $B .^{16}$

Given the promotion policies $z^{U B}$ and $z^{*}$, we can define transition functions $M^{U B}(m)$ and

\footnotetext{
${ }^{15}$ If the worst type $A$ is better than the best type $B\left[s_{A}(r\right.$, $\left.m)>s_{B}(0, m)\right]$ so that the marginal contributions cannot be equalized, then the firm only promotes type $A$ 's and $z^{U B}(m)=r$. Similarly, if $s_{B}(r, m)>s_{A}(0, m)$ then $z^{U B}(m)=0$.

16 There are other ways to define bias in our model. For instance, one could define bias as any deviation from equal promotion rates [i.e., as $\left|z^{*}(m)-r / 2\right|$ ] since the pool of initial abilities is the same for each type. This definition produces more of a bias for the majority. Alternatively, one could define the bias as any deviation from promoting the applicant who contributes the most to long-run firm profits, including the indirect contribution from mentoring others; then there is no bias. One advantage of our definition is that it is linked to a firm's preferences about diversity. Thus a firm that biases for the minority (by our definition) would also be willing to incur other costs to facilitate minority career advancement, such as hiring diversity managers.
}

$M^{*}(m)$, which give the state achieved in period $t+1$ as a function of the period $t$ state $m$ :

$$
\begin{gathered}
M^{U B}(m)=m(1-r)+z^{U B}(m) . \\
M^{*}(m)=\left\{m^{\prime} \mid m^{\prime}=(1-r) m+z,\right. \\
\text { where } \left.z \in z^{*}(m)\right\} .
\end{gathered}
$$

Figure 1 plots the surplus functions, $s_{A}(\theta, m)$ and $s_{B}(\theta, m)$, for an example. Since type $A$ is the majority $(m=0.9), s_{A}$ is greater than $s_{B}$ by the amount of the mentoring differential, in this case $\mu(0.9)-\mu(0.1)$. When unbiased promotion policies are used, by definition, the surplus of the marginal promoted employee is the same across the worker types, so that the firm promotes mostly type $A$ workers. In this example, the optimal bias is positive, so that when the optimal promotion policy is used, fewer type $A$ workers are promoted than with an unbiased promotion policy, and the marginal type $A$ worker is better than the marginal type $B$ worker.

Note that it is possible to reinterpret our model as applying to hiring rather than promotion decisions. Then the "upper level" is the firm's whole workforce, and the "lower level" is the pool of applicants. The key assumptions are that the productivity of new hires depends on 
the proportion of more senior workers with the same type and that there is a diverse applicant pool. Under this interpretation, our results characterize the firm's optimal hiring policy and the evolution of the diversity of the firm's entire work force.

\section{The Optimal Promotion Policy}

We begin our analysis by characterizing the direction of the optimal promotion bias. As a building block, we first characterize the effect of diversity on the profit of a firm that lives for only one period. The one-period analysis highlights the critical trade-off between maximizing the mentoring gain for one of the applicant pools and exploiting the scarce initial talent in both pools.

The optimal policy of a firm that lives just one period (i.e., $\delta=0$ ) is unbiased promotions $\left[z^{U B}(m)\right]$, since the firm does not care about future mentoring performed by promoted workers. The profit of such a firm is then

$$
\pi^{U B}(m)=\pi\left(m, z^{U B}(m)\right) .
$$

Suppressing the dependence of $z^{U B}$ on $m$, we can rewrite this as follows:

$$
\begin{aligned}
\pi^{U B}(m)= & z^{U B} \alpha \mu(m)+\left(r-z^{U B}\right) \alpha \mu(1-m) \\
& +\int_{0}^{z^{U B}} x(\theta) d \theta \\
& +\int_{0}^{r-z^{U B}} x(\theta) d \theta .
\end{aligned}
$$

By the envelope theorem, the effect of a change in the initial level of diversity is given by:

(2) $\frac{d}{d m} \pi^{U B}(m)=z^{U B} \alpha \mu^{\prime}(m)$

$$
-\left(r-z^{U B}\right) \alpha \mu^{\prime}(1-m) .
$$

Increasing the initial proportion of type $A$ workers leads to an increase of $\alpha \mu^{\prime}(m)$ in the men- toring received by the $z^{U B}$ type $A$ workers who are promoted, as well as a corresponding reduction of $\alpha \mu^{\prime}(1-m)$ for the $r-z^{U B}$ type $B$ workers who are promoted. Thus, the sign of $d \pi^{U B}(m) / d m$ depends on the curvature of the $\mu$ function as well as the relative proportions of type $A$ and $B$ workers promoted.

If the mentoring function is concave, then $\mu^{\prime}(1-m)>\mu^{\prime}(m)$, and increases in diversity (i.e., decreases in $m$ ) increase the mentoring of minority employees more than they reduce the mentoring of majority employees. However, since the mentoring function is nondecreasing, majority types receive more mentoring than minority types, and a myopic firm promotes more of the majority $(A)$ types than the minority $(B)$ types $\left(z^{U B}>r / 2>r-z^{U B}\right)$; thus, profits are more sensitive to the mentoring of $A$ types than $B$ types.

As a result, the firm's profit increases with the diversity of its upper level only if the mentoring function is concave and the degree of concavity is sufficient to overcome the larger weight placed on mentoring outcomes for majority workers. We shall say that sufficient concavity (SCV) holds at $m>1 / 2$ if $(d /$ $d m) \pi^{U B}(m)<0$, that is, if

$$
\mu^{\prime}(m)<\frac{r-z^{U B}(m)}{z^{U B}(m)} \mu^{\prime}(1-m) .
$$

Further, we say that sufficient concavity holds everywhere (SCV everywhere) if SCV holds for all $m>1 / 2$. Similarly, we shall say that sufficient concavity holds nowhere (SCV nowhere) if SCV fails (with a strict inequality) for each $m \geq 1 / 2$.

Now consider a firm that lives three periods. In the second period, the bias favors the minority if and only if SCV holds: SCV determines whether having more minority workers in the final period increases or decreases profits in that period. However, the problem for the firm in the first period is more subtle. A critical feature of our setting is that

$$
\begin{aligned}
& \frac{\partial^{2}}{\partial m \partial z} \pi(m, z) \\
& \quad=\alpha \mu^{\prime}(m)+\alpha \mu^{\prime}(1-m)>0,
\end{aligned}
$$


so that in any period the returns to having more of some type in the upper level are increasing in the promotion probability of that type. This effect reinforces the bias as we move back from period 2 to period 3. Suppose that SCV holds everywhere, so that the period 2 bias favors the minority. Since more minorities are promoted in the second period than would be with unbiased promotions, the firm biases for the minority (and more so than if the horizon were only one period). Incorporating this logic into an inductive argument, we can show that the characterization of the one-period profit function carries over to our infinite-horizon model, yielding unambiguous predictions about the direction of the optimal bias.

PROPOSITION 1: (i) If SCV holds everywhere, then the value of the firm is increasing with the level of diversity $(d V / d m<0)$, and the optimal promotion policy is biased in favor of the minority for each $m$. (ii) If SCV holds nowhere, then the value of the firm is increasing as the upper level becomes more homogeneous $(d V / d m>0)$, and the optimal promotion policy is biased in favor of the majority for each $m$.

In many settings, we expect that the mentoring function should exhibit decreasing returns (i.e., $\mu$ is concave). In its most literal sense, mentoring refers to a voluntary relationship between a more experienced employee and a new hire. An increase in the proportion of upperlevel employees of a given type may allow lower-level employees who would otherwise be unmatched to find a mentor (or a more appropriate mentor). The first minorities in the upper level yield high returns by providing mentoring to those who would not otherwise have received it. At higher levels of diversity, additional upper-level minorities simply provide somewhat better mentoring possibilities to those who would have been mentored anyway. This suggests decreasing returns to mentoring as diversity increases.

However, Proposition 1 shows that diminishing returns are not sufficient for the optimal promotion policy to be biased in favor of the minority type. Even with decreasing returns, a firm may still bias its promotion decisions in favor of majority types, because they make up the bulk of those promoted as a result of their advantage in the mentoring process. Consequently, our model is consistent with heterogeneity in a firm's attitudes toward diversity even under decreasing returns. Section IV characterizes how SCV depends on other exogenous elements of the model, such as the scarcity of talent and the retirement rate.

There are, of course, functional forms for which SCV holds in some regions but not others. Then, it is possible that the firm biases in favor of the majority for some values of $m$ and in favor of the minority for others.

Example 1: Suppose $\mu(m)=m^{.11}, x(\theta)=$ $1-\theta, r=0.3$, and let $\delta$ take on one of the values $\{0,0.3,0.95\}$.

Figure 2 plots the one-period value function, $\pi^{U B}(m)$, for $m \geq 1 / 2$ for Example 1. Note that profits increase with diversity except when $m \geq$ 0.927 . Figure 3 graphs the unbiased promotion rate $\left(z^{U B}\right)$ for type $A$ applicants as well as the optimal promotion rates for $\delta=0.3$ and $\delta=$ 0.95 . The bias in favor of type $B$ workers, $b(\mathrm{~m}$; $\delta)$, is the difference between the unbiased promotion rate $(\delta=0)$ for type $A$ 's and the biased promotion rate.

Figure 3 shows that for $\delta=0.95$, the firm always biases in favor of the minority; despite the fact that the one-period value function is increasing near 1 , the firm finds it worthwhile to bias promotions toward full diversity, which maximizes the one-period profit function. In contrast, if $\delta=0.3$, the firm's optimal policy is to bias in favor of the majority in the region $[0.93,0.96]$, and in favor of the minority elsewhere. Thus, even within a single firm, attitudes toward diversity will change with the initial conditions. We further discuss the dynamics of this example in the next section.

Before proceeding, we pause to observe that the dependency of profit on diversity identified in Proposition 1 has implications not only for promotion decisions, but also for any investment decision or organizational design decision whose impact varies by type. For example, the relationship between diversity and profits affects a firm's willingness to build a strong corporate culture that favors the development of the current majority type. 


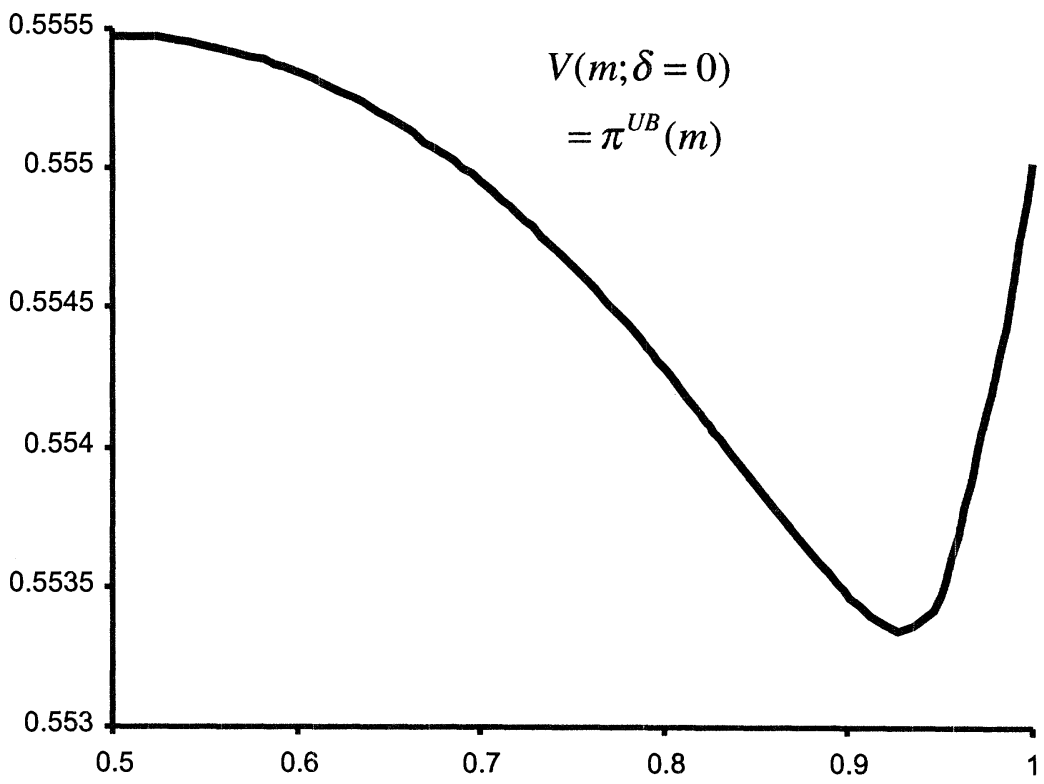

Figure 2. The Value Function with Unbiased Promotions

Note: Parameters are given in Example 1 with $\delta=0$.

\section{The Dynamics of Diversity}

We now analyze the evolution of diversity at our firm. We are interested in three questions: What is the effect of bias on the level of diversity? Is there a glass ceiling? To what extent does history matter?

We begin with some definitions. As our focus is on long-run outcomes, it is useful to define a steady-state level of diversity $m_{s}$, such that the measure of retiring $A$ managers exactly equals the measure of type $A$ 's that are optimally promoted: $r m_{s} \in z^{*}\left(m_{s}\right)$. Figure 3 graphs the "retirement function" $R(m)=\mathrm{rm}$ and the promotion policy $z^{*}(m)$ for Example 1 with $\delta=0$, 0.3 , and 0.95 . Each crossing of the retirement and promotion functions represents a steady state. Thus, $\{0.5,0.936,1\}$ are steady states when $\delta=0.3 .^{17}$ We refer to a steady state, which is always reached from a neighborhood surrounding it, as a stable steady state (e.g., the

\footnotetext{
${ }^{17}$ Steady states in our model are symmetric in that if $m$ is a steady state, so is $1-m$. Hence, in Example 1 with $\delta=$ 0.3 , the full set of steady states is $\{0,0.064,0.5,0.936,1\}$.
}

points $\{0.5,1\}$ when $\delta=0.3) .{ }^{18}$ Let $\mathscr{S} *$ be the set of stable steady states under the optimal promotion policy and let $\mathscr{S}^{U B}$ be the set of stable steady states with myopic promotions. We show in the Appendix that the level of diversity always converges to a stable steady state with either optimal (biased) or unbiased promotions, so that both $\mathscr{S}^{U B}$ and $\mathscr{S}^{*}$ are nonempty.

Type-based mentoring causes the diversity of the firm to change over time in potentially complex ways, even when promotions are unbiased. Hence, it is useful to decompose the effect of type-based mentoring on the dynamics into its direct effect on the unbiased promotion rate, and its indirect effect on the optimal bias. ${ }^{19}$ We therefore begin with results related to the stability of full diversity

\footnotetext{
${ }^{18}$ More formally, the level of diversity $m$ is stable for the transition function $M$ if there exists a $v>0$ such that, for all $v>\varepsilon>0$ such that $M(m+\varepsilon)$ and $M(m-\varepsilon)$ are single valued, $m+\varepsilon \geq(<) M(m+\varepsilon)$ and $m-\varepsilon \leq$ $(>) M(m-\varepsilon)$.

${ }^{19}$ Moreover, the dynamics of a myopic firm are of interest from a policy perspective, because they highlight the long-run outcome when firms implement a "type-blind" promotion policy.
} 


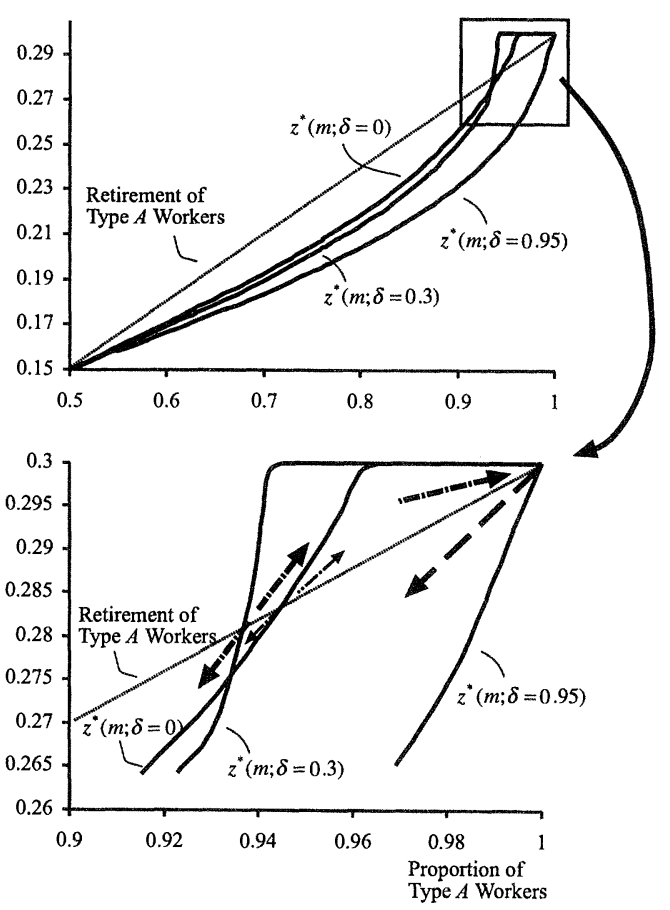

Figure 3. The Dynamic Evolution of Diversity for DifFERENT Discount FACTORS

Notes: The upper panel plots the retirement rate against the optimal promotion policies for $\delta=0,0.3$, and 0.95 . As indicated by the arrows in the lower panel, when the retirement rate is greater (less) than the promotion rate, the firm moves toward greater (less) diversity. Parameters are given in Example 1.

and the existence of multiple steady states under unbiased promotions. In general, our model is consistent with an arbitrary constellation of stable steady states. ${ }^{20}$ With some additional structure, however, it is possible to provide sharper predictions.

PROPOSITION 2: With unbiased promotions: (i) Full diversity is a stable steady state if and only if the asymmetries created by type-based mentoring are small relative to the scarcity of talent in the neighborhood of full diversity: $1 / 2 \in \mathscr{S}^{U B} \Leftrightarrow \alpha \mu^{\prime}(1 / 2)<-r x^{\prime}(r / 2)$. (ii) If

\footnotetext{
${ }^{20}$ Indeed, it can be shown that for any finite, symmetric set of points $X \subset[0,1]$, there exists a nonincreasing initial ability function $x$ and a nondecreasing mentoring function $\mu$ such that $\mathscr{Y}^{U B}=X$.
}

$\mu^{\prime \prime \prime}(m)>0 \forall m$ and $x(\theta)=\kappa-\gamma \theta$, then $\mathscr{S}^{U B} \subset\{0,1 / 2,1\}$; and if $\mu^{\prime \prime \prime}(m)<0 \forall m$ and $x(\theta)=\kappa-\gamma \theta$, then $\mathscr{S}^{U B}=\left\{m_{s}, 1-m_{s}\right\}$ for some $m_{s} \geq 1 / 2$.

We now turn to the dynamics under optimal promotions. A final definition is useful for identifying the effect of the optimal bias on the evolution of diversity.

Definition 1: Consider two sets of stable steady states, $\mathscr{Y}_{1}$ and $\mathscr{T}_{2}$. We say that $\mathscr{S}_{1}$ is more diverse than $\mathscr{S}_{2}$ if

$$
\begin{aligned}
& \min \left\{m \mid m \in \mathscr{S}_{2}, m \geq \frac{1}{2}\right\} \\
& \quad \geq \min \left\{m \mid m \in \mathscr{S}_{1}, m \geq \frac{1}{2}\right\}, \\
& \max \left\{m \mid m \in \mathscr{Y}_{2}, m \geq \frac{1}{2}\right\} \\
& \quad \geq \max \left\{m \mid m \in \mathscr{Y}_{1}, m \geq \frac{1}{2}\right\} .
\end{aligned}
$$

PROPOSITION 3: (i) If SCV holds nowhere, then $\mathscr{S}^{U B}$ is more diverse than $\mathscr{S}^{*}$, and full diversity is never a stable steady state $(1 / 2 \notin$ $\left.\varphi^{*}\right)$. (ii) If SCV holds everywhere, then $\varphi^{*}$ is more diverse than $\varphi^{U B}$ and full diversity is a stable steady state if $\alpha \mu^{\prime}(1 / 2)<-r x^{\prime}(r / 2)$.

What is the impact of bias on diversity? Not surprisingly, a bias that favors the majority (as when SCV holds nowhere) increases long-run diversity, whereas a bias in favor of the majority (as when SCV holds everywhere) decreases long-run diversity. As a result, public policies that seek to force firms to use a "type-blind" promotion policy can have ambiguous effects.

We say that the firm has a glass ceiling whenever, in the long run, the upper level is not as diverse as the (fully diverse) lower level. From Proposition 3(i), we know that full diversity is never a stable long-run outcome when the bias favors the majority. Then, firms that start with homogeneous management may diversify somewhat, but the progress of minorities always stops short of full diversity. Conversely, when the bias favors the minority [Proposition 3(ii)], full diversity is a possible long-run outcome, as long as the asymmetries created by type-based mentoring are not too large relative to the scarcity of talent.

To what extent does history matter for the 
firm? For extreme values of $\alpha$ there is a unique long-run level of diversity, whatever the historically given initial conditions.

PROPOSITION 4: For $\alpha$ sufficiently large, the unique long-run stable steady state entails a homogeneous upper level $\left(\mathscr{S}^{*}=\{0,1\}\right)$. As $\alpha$ approaches 0 , each stable steady state approaches $1 / 2 .^{21}$

When mentoring is unimportant ( $\alpha$ small), the scarcity of talent and the uniform retirement rate pushes the firm toward full diversity. Conversely, when mentoring is important ( $\alpha$ large) the upper level is necessarily dominated by one type. Then, history determines which type is dominant, but not the level of diversity.

For intermediate values of $\alpha$, interior stable steady states may arise, and more important, there may be multiple steady-state levels of diversity. We saw in Proposition 2(ii) that typebased mentoring may lead to multiple steady states with unbiased promotions; multiple steady states can arise with optimal promotions as well, as illustrated in Example 1 with $\delta=$ 0.3 . Intuitively, multiple steady states may arise because the level of minority advancement tends to be self-perpetuating: with few minorities in management, minorities are poorly mentored and hence few are promoted.

When there are multiple steady states, shortlived pressure on a firm to diversify (e.g., from legislation or other forms of social pressure) can have a long-lasting impact on the level of diversity. When pressure pushes diversity above a critical mass point, ${ }^{22}$ the gains of the minorities are self-reinforcing, even if the pressure is removed. Thus, history determines the level of long-run diversity. In Example 1 with $\delta=0.3$, a firm that starts with a homogeneous upper level stays there, unless it is forced to promote enough minorities so that $m^{t}<0.936$, in which case convergence is to full diversity.

Our theory predicts not only that the level of diversity changes over time at a firm, but that the attitude toward diversity (i.e., the direction of the bias) changes as well. In Example 1 with

\footnotetext{
${ }^{21}$ For $\mu$ concave, we have the slightly stronger result that $\mathscr{S}^{*}=\{1 / 2\}$ for $\alpha$ sufficiently small.

${ }^{22}$ By a "critical mass point," we mean a steady state that has no basin of attraction.
}

$\delta=0.3$, a firm starting just to the left of the critical mass point $m^{0}=0.936$ initially biases promotions in favor of the majority. Over time, however, diversity still increases because the optimal bias and type-based mentoring are not sufficiently strong to overcome the greater retirement rate of the majority type. As $m^{t}$ falls, the optimal bias shifts and eventually favors minorities. ${ }^{23}$

We close our discussion of dynamics with a second example that uses a "critical mass" mentoring function, which is first convex and then concave, so that mentoring is relatively ineffective until the minorities reach 30 percent of the firm. ${ }^{24}$

Example 2: $\mu(m)=0$ for $m \leq 0.3, \mu(m)=$ $0.15+0.1(m-0.3)^{.11}$ for $m>0.3, r=$ $0.3, \delta=0.3$ and $x(\theta)=1-\theta$.

Figure 4 graphs this mentoring function. ${ }^{25}$ In this example, there is a glass ceiling and multiple steady states, even though per-period profits are maximized at full diversity. Figure 5 graphs the evolution of diversity in this example from a variety of starting points. Starting from homogeneity, minorities make some initial progress into the firm, yet there is a glass ceiling: they never reach a level of representation above 10 percent, even if the firm starts out with close to 20 percent minorities. On the other hand, if external pressure pushes minorities beyond the critical mass of 20 percent, convergence is to full diversity. The region of increasing returns in the critical mass mentoring function

\footnotetext{
${ }^{23}$ Similarly, a firm starting to the right of $m^{0}=0.936$ initially biases promotions in favor of the majority, but as homogeneity increases and decreasing returns become stronger, the optimal bias shifts to favor the minority.

${ }^{24}$ This mentoring function incorporates the idea that $\mu(m)$ increases slowly at first as a result of the relative ineffectiveness of mentors who are themselves highly isolated in the organization. Mentoring becomes effective only when a critical mass of mentors is reached. Similar curvature could also arise if the efficiency of communication and information sharing rises sharply once a critical mass is reached.

${ }^{25}$ Notice that this mentoring function has an upward jump, which strictly speaking violates our smoothness assumption; however, the smoothness assumption is not necessary for a computational analysis, and in any event the function could be approximated with a differentiable function.
} 


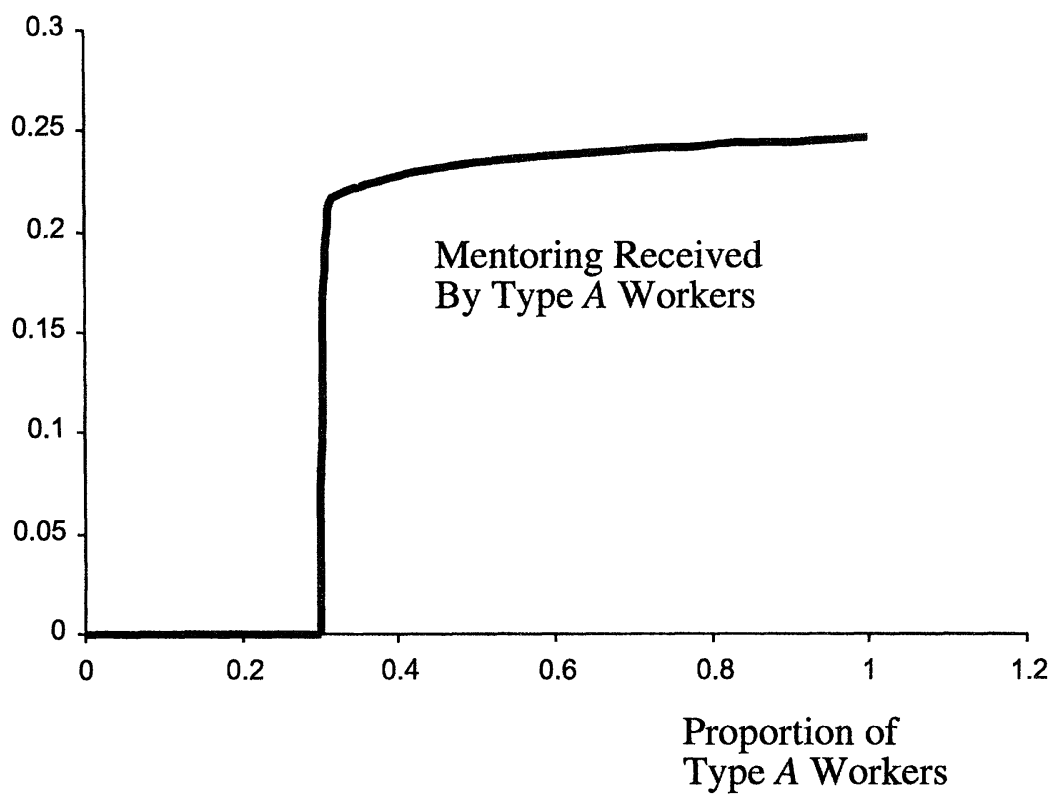

Figure 4. A Critical Mass Mentoring Function

Note: Parameters are given in Example 2.

strengthens the general tendency for minority gains to be self-perpetuating and hence for history to matter.

\section{Empirical Implications of the Model}

We now develop the empirical implications of our theory. Section IV, subsection A, identifies the exogenous parameters that lead firms to prefer diversity, and thus favor minority groups in their optimal promotion policies. Section IV, subsection B, derives comparative statics for long-run levels of diversity.

\section{A. Factors Influencing the Promotion Bias}

Section II linked a firm's attitude toward diversity to the curvature of the mentoring function, and more specifically to the SCV condition. Any factor that influences the unbiased promotion policy has an impact on whether SCV holds, and thus on the sign of the optimal promotion bias. In this section, we focus on the effects of the retirement rate $r$, the importance of mentoring $\alpha$, and the scarcity of initial ability.

We parameterize the scarcity of initial ability by $\gamma$, where an increase in $\gamma$ makes the initial ability function $x$ steeper. Formally, we assume that $x(z ; \gamma)-x(r-z ; \gamma)$ is nonincreasing in $\gamma$ for $z>r / 2$. Thus, the higher the $\gamma$, the greater the cost (in terms of lower average initial ability of promoted employees) of deviating from the policy of promoting an equal number of each type. The linear initial ability function $x(\theta ; \gamma)=\kappa-\gamma \theta$ satisfies this requirement.

Proposition 5 identifies the effects of $\alpha, r$, and $\gamma$ on both the unbiased promotion policy and on $(d / d m) \pi^{U B}(m)$, whose sign determines whether SCV is satisfied.

PROPOSITION 5: For $m>1 / 2$, (i) The unbiased promotion rate, $z^{U B}(m ; \alpha, \gamma, r)$, is (a) nonincreasing in $\gamma ;(b)$ nondecreasing in $\alpha$. (ii) $(d / d m) \pi^{U B}(m ; \alpha, \gamma, r)$ is (a) nonincreasing in $\gamma ;(b)$ nondecreasing in $\alpha$; and (c) nonincreasing in $r$ whenever $x(\theta ; \gamma)=\kappa-\gamma \theta$ and $\mu$ is concave.

An increase in the scarcity of initial ability $(\gamma)$ makes it more likely that the firm biases for the minority. As scarcity rises, it becomes more costly to promote disproportionately from the 


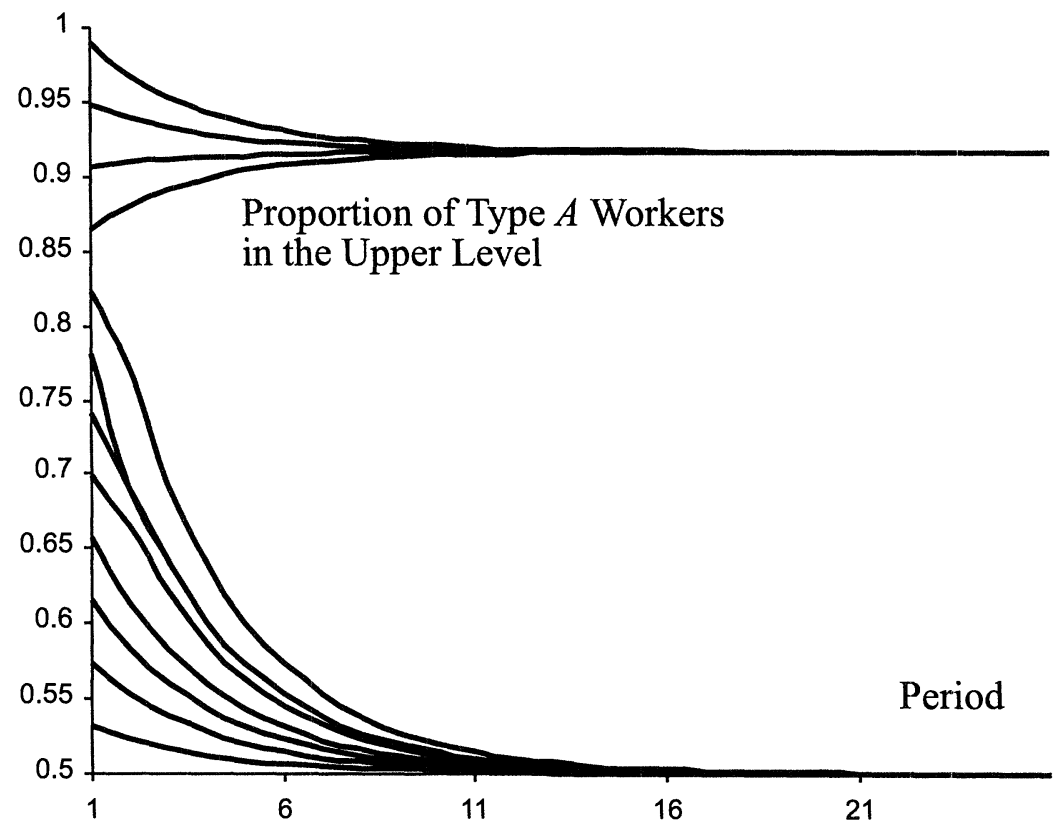

Figure 5. The "Glass Ceiling"

Notes: The figure plots the state of the firm as a function of time, starting from different initial conditions. Parameters are given in Example 2.

majority pool. The more minorities promoted with the unbiased policy, the more likely it is that a myopic firm (and, by Proposition 1, a forward-looking firm) benefits from having a diverse upper level. Industries in which scarcity is important include those that require specialized skills and experience, such as high-level management, as well as industries where "stars" are important, such as academics.

Conversely, the greater the importance of mentoring $\alpha$, the more majority employees are promoted by a myopic firm, since their mentoring advantage is larger, and thus the firm is less likely to bias for the minority. ${ }^{26}$ Industries in which mentoring is likely to be important relative to initial ability include services, law, academics, industries where networking and

\footnotetext{
${ }^{26}$ When $\alpha$ becomes sufficiently large, the firm's optimal policy will select only majority types (SCV will hold nowhere); for $\alpha$ sufficiently close to zero, unbiased promotion selects almost the same number of majority and minority types, and then the concavities of $\mu$ and SCV are (approximately) equivalent.
}

information sharing play an important role, and industries where apprenticeships are important components of training. In contrast, mentoring will be less important in industries in which jobs are well defined, require few specialized skills, and involve little information sharing.

An increase in the retirement rate $r$ has an ambiguous effect on the sign of the optimal bias. As turnover increases, the firm generally promotes more workers of both types, but longrun diversity depends on the proportion of minorities who are promoted. Suppose that the mentoring function is concave. Then, the direction of any change depends on the curvature of the initial ability function. When the initial ability function is linear, an increase in turnover increases unbiased majority and minority promotions by the same amount. But since there is a higher marginal return to minority mentors, this change makes it relatively more attractive to promote minorities. Industries with low turnover at the senior level relative to the size of the firm might include law, academics, businesses organized by partnership, and large 
bureaucracies; high turnover occurs in many high-technology industries, as well as intermediate levels of hierarchies.

\section{B. Factors Influencing Long-Run Diversity Levels}

We now explore the extent to which the comparative statics in the previous section apply to long-run levels of diversity. Since it may be difficult to directly observe the direction of the promotion bias, the results in this section may be more useful for interpreting observed patterns of diversity in firms. We take a local approach to comparative statics, in that we look at the effect of small changes in a parameter on a given steady state. ${ }^{27}$ Large changes in a parameter can alter the set of steady states, and hence are harder to analyze. Despite the fact that the local approach is somewhat restrictive, we still find that several of our results from Proposition 5 must be qualified when applied to long-run diversity levels.

Most of our results follow from the first-order conditions (FOC) for an optimal promotion policy at a stable steady state:

LEMMA 1: For all $m_{s} \neq 1 / 2$ which are stable steady states, there is a unique optimal promotion policy, $z^{*}\left(m_{s}\right)$. An interior steady state $m_{s} \notin\{0,1 / 2,1\}$ satisfies the following firstorder condition

$$
\begin{aligned}
& F O C_{s}\left(m_{s}\right)=\frac{\partial}{\partial z} \pi\left(m_{s}, z_{s}\right) \\
& +\frac{\delta}{1-(1-r) \delta} \frac{\partial}{\partial m} \pi\left(m_{s}, z_{s}\right)=0
\end{aligned}
$$

$$
\text { where } z_{s}=r m_{s} \text {. }
$$

That is, the return to promoting more majoritytype workers today is balanced against the

\footnotetext{
${ }^{27}$ Another approach would be to derive comparative statics on the optimal bias, and use these results to analyze changes in steady states. However, in contrast to the sign of the optimal bias, there is no necessary connection between comparative statics on the magnitude of the bias from a two-period model (which would follow from Proposition 5), and comparative statics on the magnitude of the bias in the infinite-horizon model.
}

(weighted) return to having a lower level of diversity in the next period. Proposition 6 identifies the main comparative statics results.

PROPOSITION 6: Each stable steady state $m_{s} \geq 1 / 2$ is:

(i) Nonincreasing in response to a small increase in $\gamma$.

(ii) Nonincreasing (nondecreasing) in response to a small increase in $\delta$ when SCV holds everywhere (nowhere).

(iii) (a) Nondecreasing in response to a small increase in $\alpha$, when SCV holds nowhere.

(b) Nondecreasing in $\alpha$ when $\delta$ is sufficiently small.

(iv) (a) Nonincreasing in response to a small increase in $r$ when SCV holds everywhere and $x(\theta)=\kappa-\gamma \theta$. (b) Nonincreasing (nondecreasing) in $r$ if $\partial z^{U B} / \partial r<(>) m_{s}$ when $\delta$ is sufficiently small.

We now look at the intuition for each of the comparative static results, as well as some of the implications. In developing the intuition it is useful to note that $M^{*}(m)=M^{U B}(m)-b(m)$, so that the effect of a parameter can be decomposed into its direct effect on the unbiased dynamics and an indirect effect resulting from changes in the optimal bias.

Proposition 6(i) shows that long-run diversity increases with (small) increases in the scarcity of ability. By Proposition 5, the direct effect of an increase in scarcity is an increase in the unbiased promotion rate for minorities. There is also an indirect effect: when the firm anticipates promoting more minorities in the future, it cares more about their mentoring and hence the bias moves toward the minority. Thus, both the direct and indirect effects support an increase in diversity.

Although we do not formally model asymmetries in initial ability between types, it is straightforward to analyze asymmetries using this result. If the initial ability of one type becomes less scarce, the promotion rates and bias shift in favor of that type. One implication is that the entry of more women and minorities into the workforce will cause firms to shift their optimal bias toward these groups. Another implication is that in a multilevel organization, 
diversity might fall in higher levels of the hierarchy. This follows because inequities in one level will lead to lower promotion rates for the disadvantaged type, and thus inequities at one level will be reinforced at the next-higher level. This is consistent with the evidence cited in the introduction about the glass ceiling, as well as Bartlett's (1997) description of diversity in the economics profession. ${ }^{28}$

Now consider the effect of changes in the discount rate. As $\delta$ increases, the firm is more willing to bias promotions to improve future mentoring. Hence, the effect of a change in $\delta$ depends on the direction of the optimal bias. If the bias favors the minority (majority), then diversity increases (falls).

As an application of this result, suppose that the owners of the firm have a larger discount rate than do the managers who make promotion decisions. In practice, this type of agency problem might arise because managers take into account the fact that they will leave the firm (or at least their current job) with positive probability in the future. Then, a firm with a mentoring function that is SCV everywhere may have a suboptimal level of diversity. Inefficiencies of this kind are consistent with the observation that many firms have internal rules or policies that restrict decisions about diversity in hiring and promotion. ${ }^{29}$ Furthermore, since such agency problems may exist even in top management, shareholders may wish to constrain the decisions of top management about diversity. ${ }^{30}$

\footnotetext{
${ }^{28}$ Notice that our finding contrasts with a simple but plausible alternative theory, whereby firms use type-blind hiring policies in response to government regulation, but then discriminate (perhaps because of a taste for discrimination) in internal promotions. The alternative theory would predict a sharp fall in diversity from the entry level to the next-higher level, but relatively constant promotion rates after that.

${ }^{29}$ Our theory also raises the possibility that some firms with agency problems might want to adopt rules that limit diversity. We do not typically observe such rules, probably because of legal and social costs associated with explicit discrimination against minorities.

${ }^{30}$ The ability of shareholders to dictate diversity policy has been a topic of recent debate by the Securities and Exchange Commission (SEC) (see Steven Wallman, "Equality Is More Than 'Ordinary Business,'" New York Times, March 30, 1997 p. 3). In 1993, the SEC reversed a long-standing policy by allowing Cracker Barrel Old Country Store to exclude a shareholder resolution that prohibited job discrimination on the basis of sexual orientation, argu-
}

The effect of a small increase in the importance of mentoring can be ambiguous because the indirect effect can oppose the direct effect. The direct effect of an increase in $\alpha$ is that more majority types are promoted by a myopic firm. In terms of the indirect effect, this will further tend to shift the optimal bias of a forward-looking firm toward the majority. However, any increase in the importance of mentoring also makes the firm more willing to bias promotions (in either direction) to optimize future mentoring. If the bias is for the majority (as when SCV holds nowhere), then the two indirect effects work in the same direction, and diversity unambiguously falls with $\alpha$. Similarly, if $\delta$ is sufficiently small, the optimal bias is small, and the direct effect dominates. Since for sufficiently large $\alpha$, SCV holds nowhere, shifts when $\alpha$ is already large should unambiguously decrease diversity.

The direct effect of a change in $r$ is already ambiguous, as we saw in Proposition 5. Of course, for $\delta$ small, the direct effect dominates. In general, increases in $r$ make the firm more willing to bias promotions (in either direction) since current promotions have an immediate and large effect on diversity. Thus, even when the initial ability function is linear (and thus faster turnover increases the returns to minority promotions under the unbiased policy), unambiguous predictions about steady states follow only when the bias favors the minority.

Although these results provide predictions about the link between exogenous features of the environment and patterns of diversity, they also require some qualification. First, note that the results for $\alpha$ and $r$ require additional assumptions: the parameters affect both the unbiased promotion rates and a firm's willingness to bias promotions. On balance, though, it seems plausible that diversity should be lower in industries in which mentoring is very important, and higher in high-turnover industries (if the mentoring function is sufficiently concave). A second issue is that, as in any model with

\footnotetext{
ing that diversity policy fell within the range of "ordinary business matters," which by SEC rules cannot be dictated by shareholder resolutions. Recently, shareholder interest groups have questioned the policy change. Our analysis suggests that shareholders may indeed have an interest in directing company policies toward diversity, even beyond the more widely cited issues of legal liability.
} 
multiple steady states, it can be difficult to draw inferences from cross-sectional observations. Two firms with the same technology operating in similar labor markets can potentially have very different levels of diversity, depending on their histories. These firms might also have very different attitudes toward diversity.

\section{Markets for Entry-Level Workers}

Our model abstracts from labor-market considerations to focus on the evolution of diversity at a single optimizing firm with a given level of diversity among its entry-level workers. In reality, the diversity of a firm's entry level is endogenous. An important question, then, is whether lower-level diversity is consistent with our assumption of type-based mentoring. Our working paper (Athey et al., 1998) considers a simplified extension of the model to include two myopic firms and a competitive labor market for entry-level employees. We now briefly outline the main insights from this extension.

We find that there may be entry-level diversity when both firms have the same majority type, even if their existing levels of diversity may differ. Then, both firms want to hire the same type, but the less diverse firm is more effective at mentoring them. Despite this asymmetry, each firm may end up with a diverse lower level, because there are decreasing returns to hiring the majority type. To understand the source of decreasing returns, observe that the more majority workers a firm hires, the more intense the competition for promotion and the lower the probability that any given majority worker is promoted. Since firms are trying to maximize the ability of promoted workers, the declining promotion probability for majority workers leads to decreasing returns. Like any scarce resource with decreasing returns, it can be optimal to share that resource across firms, which in our case means diverse entry levels.

Heterogeneity of firms (for example, in location, culture, or specialty) also tends to support entry-level diversity. We find in our working paper that the addition of a productivity benefit (unrelated to type or ability) from matching firms to workers leads to even stronger results. We allow half of the workers to be more productive at firm 1 by an amount $c \geq 0$ (observable prior to the hiring decision), whereas the other half of the workforce is more productive at firm 2 by the same amount $c$. For any $c>0$, we find that the entry level is fully diverse if the initial diversity levels of the firms are sufficiently similar.

Of course, competition could yield segregated outcomes for some parameters, in particular if the majority type differs across firms. In practice, we do sometimes observe segregated outcomes. Construction companies in some U.S. cities are dominated by a particular ethnic group. Some academic disciplines have large representations from particular nationalities, and gender integration is surprisingly heterogeneous across disciplines, even within the sciences.

In summary, the extension suggests that the insights of our single-firm model may be most applicable in industries where one type has historically dominated the industry and the existing firms differ in some way. In such industries, if the firms start out sufficiently similar and the discount rate is sufficiently low, our single-firm analysis will apply to the multifirm problem without modification. ${ }^{31}$

\section{Ex Ante Human-Capital Investment}

From a policy perspective, it is important to consider the interaction between human-capital investment decisions and firm promotion decisions when there is type-based mentoring. Anticipating poor mentoring prospects and hence a low probability of promotion even if their initial ability is high, $B$-type workers might choose to invest little in the specialized human capital required for upper-level work. Then, investments in human capital provide an additional mechanism to sustain self-perpetuating beliefs. In many cases, we expect to find one equilibrium where $B$ types have a low level of investment, and another equilibrium with a high level of investment.

These low-investment equilibria could be in-

\footnotetext{
${ }^{31}$ In Athey et al. (1998), we also compare the dynamics of the two-firm extension to those in the single-firm model with $\delta=0$. We find that the stable steady states of the single-firm model are also symmetric steady states of the two-firm model, and further, for $c>0$, the firms converge to these steady states if they start out sufficiently close. For $c$ sufficiently small, we find that full segregation is also a possible steady state.
} 
efficient for a variety of reasons. For example, as new workers will tend to invest in human capital for industries in which their type has high representation, there could be an inefficient clustering of minorities in only a few industries, occupations, or firms, when some of those minorities might have been better matched elsewhere. In fact, such behavior has been observed historically, as documented in the literature on "sex segregation" (Andrea Beller, 1984; Barbara Bergmann, 1989). Further, if one type is the minority across many industries or firms, that type might have very low incentives for human capital investment. This suggests a rationale for government policy to promote diversity where the government seeks to coordinate the economy on a high-investment equilibrium, perhaps by subsidizing education or training for type $B$ workers in certain industries.

\section{Conclusion}

This paper develops a novel perspective on discrimination and diversity. We begin with the view that firms possess a stock of upper-level employees. With type-based mentoring, the diversity of this stock matters, since it affects the flow of payoffs from newly promoted workers. A firm faces a trade-off in its promotions between homogeneity, which maximizes the mentoring of a particular type, and diversity, which allows the firm to make use of scarce talent of workers of both types. The firm's promotion policies are thus biased, in the sense that firms sacrifice current profit to increase or decrease future diversity. We find that a firm's optimal bias may favor diversity or homogeneity, and that the firm's attitude toward diversity depends on the curvature of the mentoring function, the strength of type-based mentoring, the scarcity of talent, the rate of retirement, and the discount rate. Thus, our model provides some insight as to why some firms adopt policies of affirmative action, whereas others oppose such policies and even pass over minorities who have overcome the mentoring disadvantage.

Further, our model has implications for the evolution of diversity over time, given initial conditions. Our model can exhibit a glass ceiling, whereby the proportion of minorities in the upper level reaches a stable steady state, which involves less diversity than at the lower level.
We also demonstrate that there may be more than one possible long-run level of diversity at a given firm. Even if full diversity is the most profitable steady state for a firm, it may not be optimal for a historically homogeneous firm to sacrifice immediate profits to achieve full diversity without outside pressure.

Our model is predicated on several assumptions, notably that firms select from a lowerlevel workforce with talented workers of both types, and that firms capture some of the surplus from mentoring. Our model is most likely to be applicable for industries where firm-specific human capital is important and difficult to acquire through formal education (so that mentoring is important); and where specialized or unusual skills are required and where talents vary with the firm-worker match (so that there is heterogeneity in the lower level). Examples that are especially appropriate include large industrial companies, investment banks, academic departments and professional service firms, and partnerships including those involved in law and consulting.

Empirical evidence about mentoring and diversity requires data on the diversity of organizations, preferably over time. Unfortunately, much of the publicly available data is collected at the level of individual workers, without identifying the employer, and thus there has not been much empirical research about diversity at the firm level. Further, it is difficult to identify a worker's place in the hierarchy of a firm from such data sources. Our study thus motivates the exploration of alternative data sources. For example, the data on partnership decisions by individual law firms is publicly available, and it would be especially appropriate for investigating the importance of mentoring effects. ${ }^{32}$ More broadly, if firm-level data were available for a set of firms with similar hierarchical structures, it would be interesting to examine how a variety of individual outcomes (including promotions, hiring, and turnover) depend on the type composition of the firm at different levels of the hierarchy.

Our model can be extended in a number of

\footnotetext{
${ }^{32}$ Unfortunately, the incidence of female or minority partners in law firms was very low during the 1980's and early 1990 's, so only more recent data can potentially provide sufficient variation in diversity of partners.
} 
directions. The extension in our working paper is just a start at developing a full theory of mentoring and diversity in an economy. One could add endogenous human-capital acquisition by workers, as well as the entry and exit of firms. One could develop a more detailed microeconomic model of mentoring. ${ }^{33}$ For example, one could study the incentives to build mentoring relationships, or allow the level of mentoring received by a worker to depend on the ability of the mentor. More broadly, there is the possibility to further develop a theory of the firm based on the accumulation of specialized assets over time [as in Edward Prescott and Michael Visscher (1980)]. The business strategy literature highlights a number of assets that firms build up over time, such as management talent, corporate culture, and organizational capabilities (Ingemar Dierickx and Karel Cool, 1994; Jay B. Barney, 1997). One can study various policies based on their effect on the accumulation of these assets, just as we study promotion policies based on their effect on the stock of upper-level employees. ${ }^{34}$

\section{APPENDIX}

We begin by proving two basic results about the behavior of the dynamic process. Lemma 2 demonstrates that the value function is well defined. Lemma 3 demonstrates the existence of a steady state for the unbiased and optimal promotion policies. We then proceed to prove the remaining results, following the order of the results in the text.

LEMMA 2: The value function defined by equation (1) is unique and continuous. The policy correspondence $z^{*}$ is compact valued and upper semicontinuous. $V$ and $\left|z^{*}(m)-r / 2\right|$ are both symmetric about $m=1 / 2$.

\section{PROOF:}

In the notation of Nancy Stokey and Robert Lucas (1989), our one-period payoff function,

\footnotetext{
${ }^{33}$ See Belle Rose Ragins (1997) for a discussion of this point.

${ }^{34}$ Rafael Rob and Peter Zemsky (1997)-building on the general approach developed here-explore the effect of incentive policies on the evolution of a firm's "stock" of corporate culture.
}

$F\left(m, m^{\prime}\right)=\pi\left(m, m^{\prime}-(1-r) m\right)$ is bounded and continuous; the function $\Gamma(m)=[(1-$ $r) m,(1-r) m+r]$, which characterizes the feasible values of the state variable in the next period, is nonempty, compact valued, and continuous; and the discount rate is in $(0,1)$. Hence their Theorem 4.6 holds for our model and the value function is unique and continuous and the policy correspondence is upper semicontinuous. The symmetry in $V$ and $\left|z^{*}(m)-r / 2\right|$ follow from the symmetry of our model.

LEMMA 3: $(i) M^{U B}(\cdot)$ is a single-valued, nondecreasing continuous function, and $M^{U B}(m) \geq 1 / 2$ for $m>1 / 2$.

(ii) $M^{*}(\cdot)$ is upper semicontinuous, and except for upward jumps, it is a single-valued, nondecreasing, and continuous function. Further, $M^{*}(m) \geq 1 / 2$ for $m>1 / 2$.

(iii) With either biased or unbiased promotions, the diversity of the firm converges to a steady state in $[1 / 2,1]$ for $m^{0}>1 / 2$.

\section{PROOF:}

(i) For $M^{U B}(\cdot)$ nondecreasing, note that $z^{U B}(m)=\operatorname{argmax}_{z \in[0, r]} \pi(m, z)$ and $\left(\partial^{2} / \partial m\right.$ d $z) \pi(m, z)>0$. Since the objective is differentiable, strictly concave, and strictly supermodular, $z^{U B}(m)$ must be unique everywhere, and strictly increasing when $0<z^{U B}(m)<r$. (ii) Consider the following expression for $M^{*}(m)$

$$
\begin{aligned}
M^{*}(m)= & \underset{m^{\prime} \in \Gamma(m)}{\operatorname{argmax}} \pi\left(m, m^{\prime}-(1-r) m\right) \\
& +\delta V\left(m^{\prime}\right),
\end{aligned}
$$

where $\Gamma(m)=[(1-r) m,(1-r) m+r]$. Note that the preceding objective is strictly supermodular in $m$ and $m^{\prime}$ since

$$
\begin{aligned}
& \frac{\partial^{2} \pi\left(m, m^{\prime}-(1-r) m\right)}{\partial m \partial m^{\prime}} \\
& =\mu^{\prime}(m)+\mu^{\prime}(1-m) \\
& \quad-x^{\prime}\left(m^{\prime}-(1-r) m\right) \\
& \quad-x^{\prime}\left(r-m^{\prime}-(1-r) m\right)>0 .
\end{aligned}
$$

Further, $\Gamma(m)$ is nondecreasing in the strong set order (see Paul Milgrom and Christina Shan- 
non, 1994). Hence, $M^{*}(m)$ is nondecreasing and single valued everywhere except at upward jumps. Thus, every selection from $M^{*}(m)$ is a continuous function but for upward jumps. (iii) Clearly, for $m>1 / 2, M^{U B}(m) \geq 1 / 2$. Consider $m^{\prime}<1 / 2$ and $m>1 / 2$. Together, condition (A1) and the symmetry of $V$ implies that $\pi\left(m, m^{\prime}-(1-r) m\right)+\delta V\left(m^{\prime}\right)<\pi(m, 1-$ $\left.m^{\prime}-(1-r) m\right)+\delta V\left(1-m^{\prime}\right)$, which establishes that for $m>1 / 2, M^{*}(m) \geq 1 / 2$ for $m>1 / 2$. By our constraint, $M^{*}(m) \leq 1$ for $m>1 / 2$. Hence, following Milgrom and John Roberts (1994), starting from $m^{0}>1 / 2$, a fixed point of $M^{*}$ and $M^{U B}$ exists on $[1 / 2,1]$.

\section{PROOF OF PROPOSITION 1:}

We proceed by induction on number of periods remaining in a firm's life. We know that when there is 1 period to go, the value function is equal to $\pi^{U B}(m)$, which is nonincreasing in $m \geq 1 / 2$ when SCV holds everywhere. Consider the problem with $T$ periods to go, and assume that the value of the firm with $T-1$ periods to go, $V^{(T-1)}(m)$, is nonincreasing in $m$. The firm then solves

$$
\begin{aligned}
V^{(T)}(m) & =\max _{z}\{\pi(m, z) \\
& \left.+\delta V^{(T-1)}((1-r) m+z)\right\}
\end{aligned}
$$

Observe that $\left(\partial^{2} / \partial m \partial z\right) \pi(m, z) \geq 0$. Since $V^{(T-1)}(m)$ is nonincreasing, clearly $z^{(T)}(m) \leq$ $z^{U B}(m)$, and so $(\partial / \partial m) \pi\left(m, z^{(T)}\right) \leq(\partial /$ $\partial m) \pi\left(m, z^{U B}\right) \leq 0$. Using this and the envelope theorem, $(d / d m) V^{(T)}(m)=(\partial / \partial m) \pi(m$, $\left.z^{(T)}\right)+\delta \cdot(1-r) V^{(T-1)^{\prime}}\left((1-r) m+z^{(T)}\right) \leq 0$. By induction, and since monotonicity is preserved by infinite sums, the infinite-horizon value function is also nonincreasing in $m \geq 1 / 2$. The argument is analogous for SCV holds nowhere.

\section{PROOF OF PROPOSITION 2:}

(i) $1 / 2 \in \mathscr{Y}^{U B}$ if and only if $z^{U B^{\prime}}(1 / 2) \leq r$. Using the implicit function theorem, $z^{U B^{\prime}}(1 / 2)=$ $-\alpha \mu^{\prime}(1 / 2) / x^{\prime}(r / 2)$; substituting gives the result. (ii) Suppose $x(\theta)=\kappa-\gamma \theta$. Since $M^{U B}(m)=(1-$ r) $m+z^{U B}(m)$, the curvature of $M^{U B}$ follows that of $z^{U B}$. For $z^{U B}<1, z^{U B}(m)=1 / 2 r+\alpha(\mu(m)-$ $\mu(1-m)) /(2 \gamma)$. Thus, $\left(\partial^{2} z^{U B} / \partial m^{2}\right)=\alpha\left(\mu^{\prime \prime}(m)-\right.$ $\left.\mu^{\prime \prime}(1-m)\right) /(2 \gamma)$. Hence, if $\mu^{\prime \prime \prime}(m)>0$ for all $m$, then $M^{U B}$ is concave over $(1 / 2,1]$, and there is at most one stable steady state in this interval; the steady state exists if and only if $M^{\prime}(1 / 2)>1$. If $\mu^{\prime \prime \prime}(m)<0$ for all $m$, then $M^{U B}$ is convex over $(1 / 2$, $1]$, and there are at most two steady states in the interval, and only state $m=1$ can be stable.

\section{PROOF OF PROPOSITION 3:}

The rankings of $\mathscr{S}^{*}$ and $\mathscr{S}^{U B}$ follow by Milgrom and Roberts (1994), using our results on the sign of the bias from Proposition 1. Now consider the question of whether full diversity is a stable steady state. Recall that $z^{U B}(1 / 2)=r / 2$. Hence, $z^{*}(1 / 2)=z^{U B}(1 / 2)+b(1 / 2)=r / 2$ if and only if $b(1 / 2)=0$, which is the case when SCV holds everywhere, but not when SCV holds nowhere. In the case where SCV holds everywhere, the bias favors the minority, and so if $1 / 2$ is stable under unbiased promotions, it will also be stable with optimal promotions.

\section{PROOF OF PROPOSITION 4:}

For $\alpha$ sufficiently large, $z^{U B}=r$ and $\mathscr{S}^{U B}=$ $\{0,1\}$. Further, for $\alpha$ sufficiently large SCV holds nowhere and the bias is for the majority. Hence, by Proposition $3 \mathscr{S}^{*}=\{0,1\}$ as well. As $\alpha \rightarrow 0, z^{U B} \rightarrow r / 2$ and $b(m) \rightarrow 0$ and hence $M^{*}(m) \rightarrow m(1-r)+r / 2$ so that $\varphi^{*} \rightarrow\{1 / 2\}$. If $\mu$ is concave, then SCV is satisfied everywhere for $\alpha$ sufficiently small, and from Proposition 2 (ii) the stable steady state in the neighborhood of $1 / 2$ is $1 / 2$.

\section{PROOF OF PROPOSITION 5:}

Consider first the parameter $\gamma$. Recall that $z^{U B}(m ; r, \alpha, \gamma)=\operatorname{argmax}_{z \in[0, r]} \pi(m, z ; r, \alpha$, $\gamma)$, and that $(\partial / \partial z) \pi=x\left(z^{U B} ; \gamma\right)-x(r-$ $\left.z^{U B} ; \gamma\right)+\alpha[\mu(m)-\mu(1-m)]$. By definition, $x\left(z^{U B} ; \gamma\right)-x\left(r-z^{U B} ; \gamma\right)$ is nonincreasing in $\gamma$ for $z^{U B} \geq r / 2$, which holds if $m \geq 1 / 2$. Thus, $\left(\partial^{2} / \partial z \partial \gamma\right) \pi \leq 0$, and $z^{U B}$ must be nonincreasing in $\gamma$. But then, $z^{U B} \mu^{\prime}(m)-(r-$ $\left.z^{U B}\right) \mu^{\prime}(a-m)$ is nonincreasing in $\gamma$. Consider next the parameter $\alpha$. Since $\mu(m)-\mu(1-$ $m)>0$ for $m \geq 1 / 2,\left(\partial^{2} / \partial z \partial \alpha\right) \pi \geq 0$, which implies that $z^{U B}$ and $z^{U B} \mu^{\prime}(m)-(r-$ $\left.z^{U B}\right) \mu^{\prime}(1-m)$ are nondecreasing in $\alpha$. Finally, consider the parameter $r$ and the linear initial ability function. In this case, $(\partial /$ $\partial r) z^{U B}=1 / 2$, and so $\left(\partial^{2} / \partial r \partial m\right) \pi^{U B}=$ $1 / 2 \alpha\left[\mu^{\prime}(m)-\mu^{\prime}(1-m)\right]$. Thus, if $\mu$ is concave, $(\partial / \partial m) \pi^{U B}$ is nonincreasing in $r$. 


\section{PROOF OF LEMMA 1:}

We begin by arguing that the optimal promotion policy is unique at a stable steady state. Recall that Lemma 3 implies that $M^{*}$ is single valued almost everywhere, except at upward jumps. By definition, $r m_{s}$ is one optimal promotion policy at state $m_{s}$. Suppose that there is another optimal promotion rule, $z^{\prime}>r m_{s}$. Then there must be an upward jump in $M^{*}$ at $m_{s}$; suppose that the jump is of size $d$. But this implies that for all $d>\varepsilon>0, M^{*}\left(m_{s}+\varepsilon\right)>$ $m_{s}+d>m_{s}+\varepsilon$, contradicting the definition of a stable steady state.

Now consider an interior steady state $m_{s} \notin$ $\{0,1 / 2,1\}$ and the associated steady state promotion policy $z_{s}=r m_{s}$. Since

$$
z_{s}=\underset{z \in[0, r]}{\operatorname{argmax}} \pi\left(m_{s}, z\right)+\delta V\left(z+(1-r) m_{s}\right)
$$

it must be that $\pi_{2}\left(m_{s}, r m_{s}\right)+\delta V^{\prime}\left(m_{s}\right)=0$ wherever this derivative exists. To solve for $V^{\prime}$, let $\vec{z}=\left(z^{1}, z^{2}, \cdots\right)$, and define

$$
\begin{aligned}
\tilde{V}(m, \vec{z})= & \pi\left(m, z^{1}\right) \\
& +\delta \pi\left(z^{1}+(1-r) m, z^{2}\right)+\cdots .
\end{aligned}
$$

If $m_{s}$ is a steady state, then $V^{\prime}\left(m_{s}\right)=(d /$ $\left.d m_{s}\right) \tilde{V}\left(m_{s}, \vec{z}_{s}\right)$ where $\vec{z}_{s}=\left(r m_{s}, r m_{s}, \cdots\right)$. Вy the envelope theorem, we have that

$$
\begin{aligned}
\frac{d}{d m_{s}} \tilde{V}\left(m_{s}, \vec{z}_{s}\right) & =\sum_{t=0}^{\infty}(1-r)^{t} \delta^{t} \pi_{1}\left(m_{s}, r m_{s}\right) \\
& =\frac{\pi_{1}\left(m_{s}, r m_{s}\right)}{1-(1-r) \delta} .
\end{aligned}
$$

Inserting this into the expression $\pi_{2}\left(m_{s}\right.$, $\left.r m_{s}\right)+\delta V^{\prime}\left(m_{s}\right)=0$ gives the result.

\section{PROOF OF PROPOSITION 6:}

(i) Applying the implicit function theorem, since by assumption $m_{s}$ is optimal, it suffices to check that

$$
\begin{aligned}
& \frac{\partial F O C_{s}(m ; \gamma)}{\partial \gamma} \\
& \quad=\frac{\partial[x(r m ; \gamma)-x((1-r) m ; \gamma)]}{\partial \gamma} \geq 0
\end{aligned}
$$

for $m>1 / 2$, which holds by definition. (ii) Using

$$
\begin{aligned}
\pi_{1}\left(m_{s}, r m_{s}\right)= & x\left(r m_{s}\right)-x\left(r\left(1-m_{s}\right)\right) \\
& +\alpha \mu\left(m_{s}\right)-\alpha \mu\left(1-m_{s}\right) \\
\pi_{2}\left(m_{s}, r m_{s}\right)= & m_{s} \alpha \mu^{\prime}\left(m_{s}\right) \\
& -\left(1-m_{s}\right) \alpha \mu^{\prime}\left(1-m_{s}\right)
\end{aligned}
$$

we have that

$$
\begin{aligned}
& \frac{\partial F O C_{s}(m ; \delta)}{\partial \delta} \\
& =\frac{r \alpha\left(m \mu^{\prime}(m)-(1-m) \mu^{\prime}(1-m)\right)}{(1-(1-r) \delta)^{2}} .
\end{aligned}
$$

Consider $m>1 / 2$. If $\mu$ is globally SCV, then $z^{U B}(m) \mu^{\prime}(1-m)>\left(r-z^{U B}(m)\right) \mu^{\prime}(m)$. Then $z^{U B}(m)>z^{*}(m)=r m$ implies that $\partial F O C_{s}(m) / \partial \delta>0$. Conversely, if $\mu$ is nowhere SCV, $\partial F O C_{s}(m) / \partial \delta<0$. (iii) Observe first that

$$
\begin{aligned}
\frac{\partial}{\partial \alpha} F O C_{s}(m ; \alpha)= & \mu(m)-\mu(1-m) \\
& +\frac{r \delta}{1-(1-r) \delta}\left(m \mu^{\prime}(m)\right. \\
& \left.-(1-m) \mu^{\prime}(1-m)\right) .
\end{aligned}
$$

Suppose that SCV holds nowhere. Observe that by Proposition 1 and optimality of the stable promotion policy, SCV nowhere implies that $r m_{s}>z^{U B}\left(m_{s}\right)$. But then, the second term in $(\partial / \partial \alpha) F O C_{s}(m ; \alpha)$ must be greater than $(\partial /$ $\partial m) \pi^{U B}(m)$, which in turn is positive by SCV nowhere. For $\delta$ small, recall that $m_{s} \in \mathscr{S}^{U B}$ satisfies $z^{U B}\left(m_{s}\right)-r m_{s}=0$. Consider $m_{s} \geq$ $1 / 2$. Then the diversity of $\mathscr{S}^{U B}$ is increasing 
(decreasing) in a parameter if $z^{U B}\left(m_{s}\right)-r m_{s}$ is decreasing (increasing) in that parameter. It is straightforward to show that $\partial z^{U B} / \partial \alpha>0$. (iv) In the linear case, we have

$$
\begin{aligned}
& \frac{\partial}{\partial r} F_{O C_{s}}(m ; r) \\
& =-(2 m-1) \gamma+\frac{\alpha \delta(1-\delta)}{(1-(1-r) \delta)^{2}}\left(m \mu^{\prime}(m)\right. \\
& \left.\quad-(1-m) \mu^{\prime}(1-m)\right) .
\end{aligned}
$$

By Proposition 2 and optimality of the stable promotion policy, SCV everywhere implies that $r m_{s}<z^{U B}\left(m_{s}\right)$. But then, the second term in $(\partial / \partial r) F O C_{s}(m ; r)$ must be less than $(1 / r)(\partial /$ $\partial m) \pi^{U B}(m)$, which in turn is negative by SCV everywhere. For $\delta$ small, we can consider whether $(\partial / \partial r)\left(z_{s}^{U B}-r m_{s}\right)<0$, which holds if $\partial z^{U B} / \partial r<m_{s}$.

\section{REFERENCES}

Arrow, Kenneth. "The Theory of Discrimination," in Orley C. Ashenfelter and Albert Rees, eds., Discrimination in labor markets. Princeton, NJ: Princeton University Press, 1973, pp. 3-33.

Athey, Susan; Avery, Christopher and Zemsky, Peter. "Mentoring, Discrimination and Diversity in Organizations." Stanford GSB Working Paper No. 1317, 1994. "Mentoring and Diversity." Mimeo, Massachusetts Institute of Technology, October 1998.

Barney, Jay B. Gaining and sustaining competitive advantage. New York: AddisonWesley, 1997.

Bartlett, Robin. "Report of the Committee on the Status of Women in the Economics Profession." American Economic Review, May 1997 (Papers and Proceedings), 87(2), pp. 506-10.

Beller, Andrea. "Trends in Occupational Segregation by Sex and Race, 1960-1981," in Barbara F. Reskin, ed., Sex segregation in the workplace. Washington, DC: National Academy, 1984, pp. 11-26.

$\rightarrow$ Bergmann, Barbara. "Does the Market for Women's Labor Need Fixing?" Journal of Economic Perspectives, Winter 1989, 3(1), pp. 43-60.
Business Week. "White, Male, and Worried." January 31, 1994, pp. 50-55.

$\rightarrow$ Coate, Stephen and Loury, Glenn. "Will Affirmative-Action Policies Eliminate Negative Stereotypes?" American Economic Review, December 1993, 83(5), pp. 1220-40.

$\rightarrow$ Cornell, Bradford and Welch, Ivo. "Culture, Information and Screening Discrimination," Journal of Political Economy, June 1996, 104(3), pp. 542-72.

Dierickx, Ingemar and Cool, Karel. "Competitive Strategy, Asset Accumulation and Firm Performance," in H. Daems and H. Thomas, eds., Strategic groups and strategic moves. Oxford: Pergamon Press, 1994, pp. 63-80.

Dreher, George F. and Cox, Taylor H. "Race, Gender, and Opportunity: A Study of Compensation Attainment and the Establishment of Mentoring Relationships." Journal of Applied Psychology, June 1996, 81(3), pp. 297 308.

$\rightarrow$ Greif, Avner. "Contract Enforceability and Economic Institutions in Early Trade: The Maghribi Traders' Coalition." American Economic Review, June 1993, 83(3), pp. 525-48. $\rightarrow$. "Cultural Beliefs and the Organization of Society: A Historical and Theoretical Reflection on Collectivist and Individualist Societies." Journal of Political Economy, October 1994, 102(5), pp. 912-50.

$\rightarrow$ Ibarra, Herminia. "Homophily and Differential Returns: Sex Differences in Network Structure and Access in an Advertising Firm." Administrative Science Quarterly, September 1992, 37(3), pp. 422-47.

Jones, Del. "Setting Diversity's Foundation in the Bottom Line." USA Today, October 15, 1996, p. 4B.

Kanter, Rosabeth. Men and women of the corporation. New York: Basic Books, 1977.

$\rightarrow$ Milgrom, Paul and Roberts, John. "Comparing Equilibria." American Economic Review, June 1994, 84(3), pp. 441-59.

$\rightarrow$ Milgrom, Paul and Shannon, Christina. "Monotone Comparative Statics." Econometrica, January 1994, 62(1), pp. 157-80.

Morrison, Ann and Von Glinow, Mary Ann. "Women and Minorities in Management." American Psychologist, February 1990, 45(2), pp. 200-08. 
National Law Journal. "Women's Progress Slows at Top Firms." May 6, 1996, p. A1.

$\rightarrow$ Noe, Raymond. "Women and Mentoring: A Review and Research Agenda." Academy of Management Review, January 1988, 13(1), pp. 65-78.

Prakash, Gautam. The insider's guide to management consulting. San Francisco: Wet Feet Press, 1995.

$\rightarrow$ Prescott, Edward and Visscher, Michael. "Organization Capital." Journal of Political Economy, June 1980, 88(3), pp. 446-61.

$\rightarrow$ Ragins, Belle Rose. "Diversified Mentoring Relationships in Organizations: A Power Perspective." Academy of Management Review, April 1997, 22(2), pp. 482-521.

Rob, Rafael and Zemsky, Peter. "Cooperation, Corporate Culture and Incentive Intensity." INSEAD Working Paper Series, 1997.

Rosen, Asa. "An Equilibrium Search-Matching Model of Discrimination." European Economic Review, August 1997, 41(8), pp. 1589-613.
Rothstein, Donna. "Supervisor Gender, Race, and Ethnicity and the Labor Market Outcomes of Young Workers." Mimeo, U.S. Bureau of Labor Statistics, Washington, DC, 1997.

$\rightarrow$ Spurr, Stephen. "Sex Discrimination in the Legal Profession: A Study of Promotion." Industrial and Labor Relations Review, April 1990, 43(4), pp. 406-17.

Stokey, Nancy and Lucas, Robert. Recursive methods in economic dynamics. Cambridge, MA: Harvard University Press, 1989.

Wall Street Journal, The. "Workplace: Labor's Martin is Out to Break the Glass Ceiling." August 9, 1991, p. B1.

. "Women Make Strides, But Men Stay Firmly in Top Company Jobs." March 29, 1994, p. A1.

Wallman, Steven. "Equity Is More Than 'Ordinary Business" "New York Times, March 30, 1997, p. 3. 